IJAMSR 3 (4) www.ijamsr.com CrossRef: https://doi.org/10.31426/ijamsr.2020.3.4.3212

\title{
CERAMIC VENEERS -CASE REPORT
}

\section{Dr. Swati Arora Verma*, Dr. Neha Milani}

*BDS FAD, DR. D. Y. Patil Dental College \& Hospital, Pimpri, Pune, India.

MDS, Prosthodontics, DR. D. Y. Patil Dental College \& Hospital, Pimpri, Pune, India.

Email: drswatikkarora@gmail.com

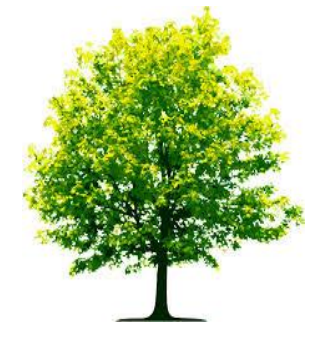

Keywords:

Ceramic Veneers,

Malalignment,

Discoloration,

Cementation

\section{A B S T RA C T}

Due to their lifelike appearance \& longevity, ceramic veneers have become a popular restorative procedure for anterior teeth. However, it is a multi-sitting process and often there are choices that the clinician faces at each step. Along with that, there are certain best practices and points to be taken care of during the treatment. This paper aims to describe the step-by-step procedure followed in one such treatment of a 32-year-old lady who was unhappy with the malalignment and discoloration of her teeth. Ceramic veneers provided an ideal solution to restore her smile to her satisfaction. Based on the clinical findings, it can be concluded that each step from diagnosis to treatment planning to mock-ups to final cementation are equally critical for the success of the procedure and ensuring patient satisfaction.

Citation: Swati Arora Verma, Neha Milani (2020). Ceramic VeneersCase Report: International Journal of Advanced Multidisciplinary Scientific Research (IJAMSR) ISSN:2581-4281, 3 (4), April, 2020, Pp 16 - 26 
IJAMSR 3 (4) www.ijamsr.com CrossRef: https://doi.org/10.31426/ijamsr.2020.3.4.3212

\section{International Journal of Advanced Multidisciplinary Scientific Research (IJAMSR) ISSN:2581-4281}

\section{Introduction:}

A dental veneer is a thin layer of composite material or dental porcelain bonded to the surface of a tooth. Veneers can be either composite or ceramic:

- Composite veneers, also known as 'same day veneers', are a less invasive way to restore the smile. If there are worn, chipped or damaged teeth then composite resin can be sculpted directly onto the teeth to produce beautiful, lifelike results.

- Ceramic Veneers are thin porcelain shields that cover only the front face of the teeth. They represent a permanent solution to change or improve the image of stained, chipped, broken or unaesthetic teeth.

Respectively, composite veneers provide good aesthetic outcome and patient's satisfaction. However, due to its physical properties and to the bonding strength when compared to porcelain veneers, composite veneers tend to fail significantly faster than porcelain veneers (Ref: [1]).
Hence, with that background ceramic veneers are an established conservative treatment option for the restoration of facial defects, fractures, malformation, or misalignment of teeth (Ref: [2]). Various clinical studies have determined successful treatments, and reviews have confirmed the clinically acceptable longevity of veneers (Ref: [3], [4], [5]).

From the result of the previous studies, it can be concluded that porcelain veneers can provide a predictable aesthetic acceptance, while composite veneers can be the treatment of choice for patients who appreciate minimally invasive approaches (Ref: [1]).

\section{Using Ceramic Veneers}

Ceramic veneers have gained popularity as a common aesthetic treatment for changing or improving the image of stained, chipped, broken or unaesthetic teeth. However, as is the case for any treatment method, there are Indications and Contra-indications on use of ceramic veneers.

\subsection{When to Use Ceramic Veneers (Indications)}

Improving the appearance in cases requiring treatment of discolored or chipped teeth. The below table captures illustrative scenarios 
IJAMSR 3 (4) www.ijamsr.com CrossRef: https://doi.org/10.31426/ijamsr.2020.3.4.3212

where ceramic veneers can prove to be the preferred treatment option (Ref: [6],[7]):

- Improve extreme discolorations tetracycline staining, fluorosis, devitalized teeth and darkening of teeth with age.

- Repair chipped or fractured teeth

- Closing of diastemas between teeth

- Lengthen anterior teeth.

There are multiple studies that have recommended the use of ceramic veneers as a more durable solution, since porcelain hardens into a durable tooth-like structure.

Below table presents the 'Magne and Belser classification' for the indication of ceramic veneers (Ref: [8]):

\section{Type-1 Teeth resistant to bleaching}

Type 1A- Tetracycline discoloration

Type 1B- Teeth unresponsive to bleaching

Type-2 Major morphological modification

Type 2A- Conoid teeth

Type 2B- Diastema or interdental triangles to be closed

Type 2C-Augmentation of incisal length or facial prominence

Type-3 Extensive restoration

Type 3A- Extensive coronal fracture

Type 3B- Extensive loss of enamel by erosion or wear

Type 3C- Generalized congenital malformation

\subsection{When to Use Other Treatments (Contra Indications)}

The scenarios where it is not recommended to use ceramic veneers are listed below (Ref: [6], [7]):

- If little or no enamel is present. In such cases, full crown should be considered.

- In case of certain tooth-to-tooth habits like bruxing or clenching, or other parafunctional habits like pencil chewing or ice crushing. The veneer treatment would not provide the long-term solution as expected.

- Cases of severe crowding of teeth

- Occlusal problems such as Class III \& end-to-end bites.

\section{Case Report - Ceramic Veneer Treatment Case}

\subsection{Case Overview}

Treatment is conducted in a 4-step process. Each step in the process is critical for the overall success of the treatment, and the clinician needs to ensure proper care and precautions are taken to ensure desired outcomes and patient satisfaction. Each of the following step is discussed in more 
IJAMSR 3 (4) www.ijamsr.com CrossRef: https://doi.org/10.31426/ijamsr.2020.3.4.3212

detail for the case in following sections of

this paper.
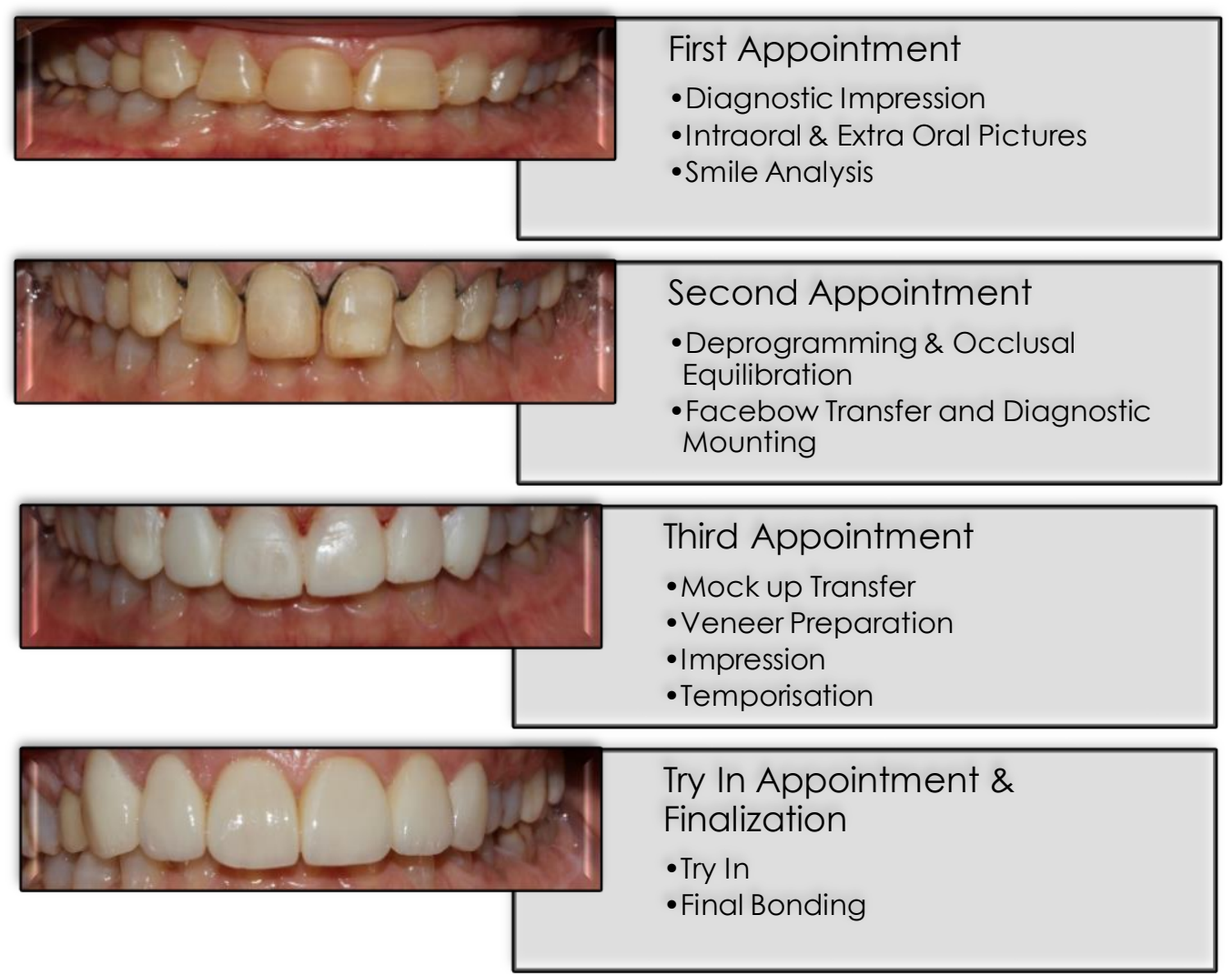

Figure 1: Case Overview

\subsection{Patient Profile}

The patient was a 32-year-old woman with discoloration \& malalignment in teeth. She reported with the malalignment of teeth and was unhappy with the color of her teeth. She wanted a Hollywood smile.

- Age: 32 years
- Gender: Female

- Chief Complaint: Patient reported with the malalignment of teeth and was unhappy with the color of her teeth. She wanted a Hollywood smile.

- Clinical Examination: She had discolored and misaligned teeth. 
IJAMSR 3 (4) www.ijamsr.com CrossRef: https://doi.org/10.31426/ijamsr.2020.3.4.3212

\section{International Journal of \\ Advanced Multidisciplinary Scientific Research (IJAMSR) ISSN:2581-4281}

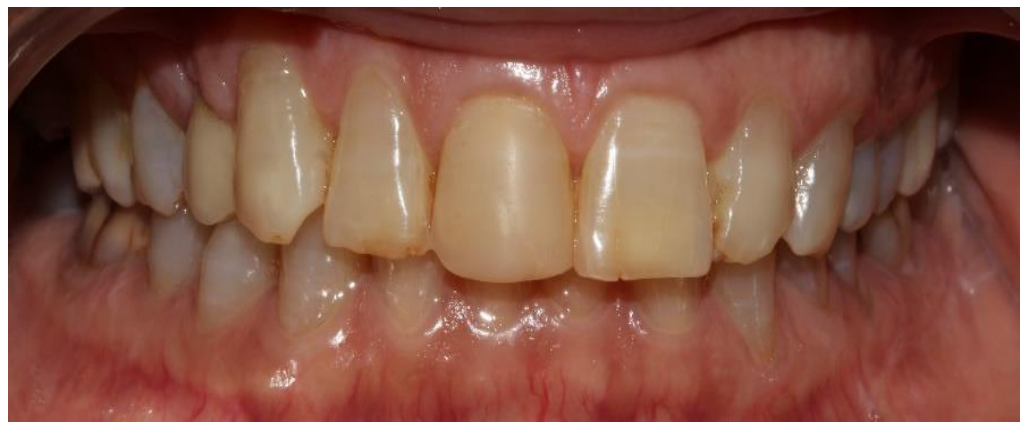

Figure 2: Pre Operative Picture (Case Review)

\subsection{Diagnosis \& Treatment Alternatives}

Diagnostic impressions were taken, suggesting treatment requiring veneers for smile design.

- Diagnosis: Teeth discoloration and smile designing.

- Treatment options: Direct composite veneers, ceramic emax veneers, indirect composite veneers.

Based on the patient requirements and current condition, use of ceramic veneers was the recommended approach. However, since ceramic veneers are an aesthetic procedure, there was a detailed discussion with the patient on the benefits, risks and procedure involved.

- Treatment Prescribed: Emax indirect veneers from 13 to 23. This was finalized based on the consideration of benefits from ceramic veneers in such a case, and the discussion with the patient.

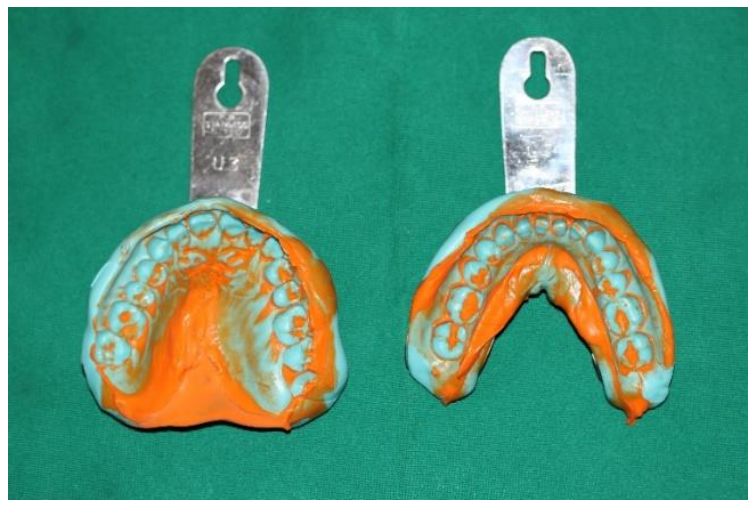

Figure 1: Diagnostic Impressions (Case Review) 
IJAMSR 3 (4) www.ijamsr.com CrossRef: https://doi.org/10.31426/ijamsr.2020.3.4.3212

\subsection{Procedure - Mock Up \& Preparation}

Mock-ups based on diagnostic impressions were used \& preparation done for treatment.

- Pre-operative pictures and impressions were recorded.

- Chairside deprogramming was carried out with Lucia Jig. After deprogramming and occlusal equilibration, impressions and facebow.
- record was made for diagnostic mounting.

- Mock-up was done post the diagnostic impressions.

- Once the patient was satisfied with the mock-up impressions, veneer preparation was started.

- Veneer preparations were done from 13 to 23. Mylar strips were used for the interproximal surfaces.

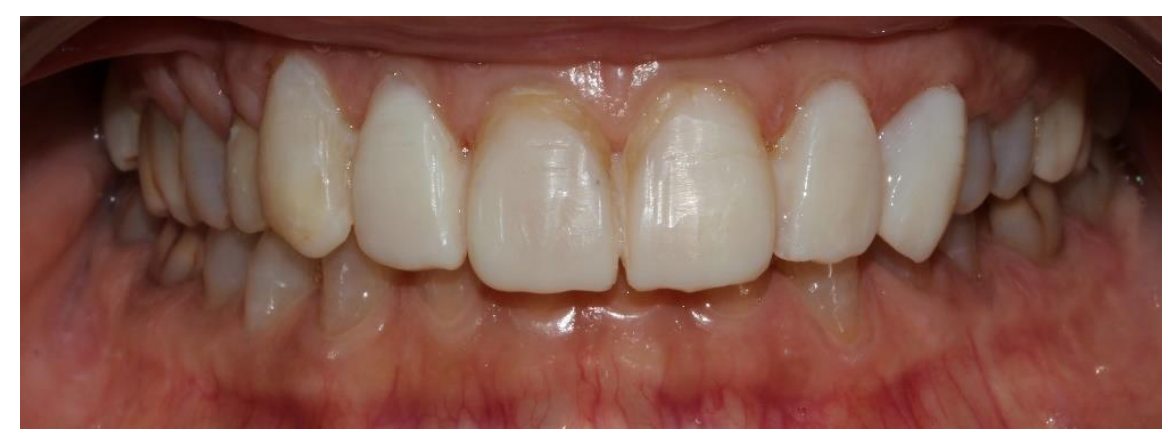

Figure 2: Mock Up (Case Review)

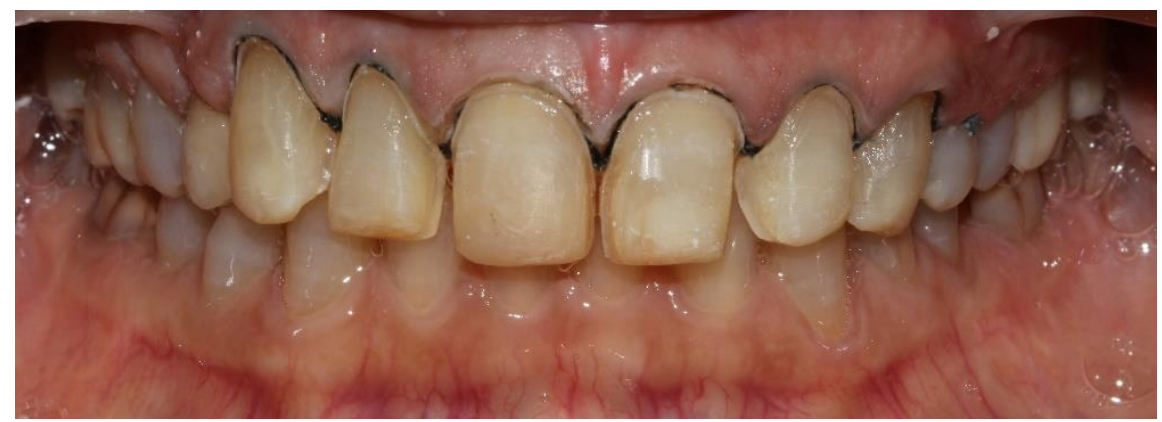

Figure 3: Preparation (Case Review) 
IJAMSR 3 (4) www.ijamsr.com CrossRef: https://doi.org/10.31426/ijamsr.2020.3.4.3212

\subsubsection{Procedure - Preparation for}

\section{Treatment}

First step of the procedure is preparing the teeth for the treatment. This is an extremely important step, as this lays the foundation of the remaining treatment, thus determining the ultimate aesthetic result and longevity of the procedure.

There are four popular types of preparations, as listed below (Ref: [9]):

a) Window preparation: In this, the incisal edge of the tooth is preserved.

b) Feather preparation: The incisal edge of the tooth is prepared, but incisal length is not reduced.

c) Bevel preparation: The incisal edge of the tooth is prepared Bucco-palatally and the length of the edge is reduced to 0.5 to $1 \mathrm{~mm}$.

d) Incisal overlap preparation: The incisal edge of the tooth is prepared Buccopalatable, and length is reduced to 2 $\mathrm{mm}$; in this case, the veneer is extended to the palatal aspect of the tooth

Further, it seems from past studies that in window preparation, the occlusal stress is highly concentrated on the incisal third which may lead to restoration getting fractured. Hence, the best support for restoration and distribution of occlusal forces over a larger surface area is provided by incisal overlap preparation. In addition, it should be noted that it is possible to achieve better incisal translucency with reduction of the incisal edge (Ref: [10], [11]).

There are four key steps for tooth preparation that were followed in this case:

a) Labial Reduction: A horizontal depth cut was applied, approximately $0.3 \mathrm{~mm}$ from one proximal line angle to the other. Post this, entire gingival margin is paralleled and a definitive chamfer finish line prepared. It is important to make sure that there are no sharp line angles.

Academic studies also support this line of treatment since the chamfer finish line allows easy access, increases the area of enamel, provides better visuality, and helps in maintaining better hygiene. The subgingival line is preferred in severe discoloration to provide the bulk of veneer thickness (Ref:[12],[13],[14]) 
IJAMSR 3 (4) www.ijamsr.com CrossRef: https://doi.org/10.31426/ijamsr.2020.3.4.3212

\section{International Journal of Advanced Multidisciplinary Scientific Research (IJAMSR) ISSN:2581-4281}

b) Interproximal Reduction: this is the process of mechanically removing enamel from between the teeth.

c) Incisal Modification: there are three common types of techniques used for incisal modification based on where the preparation is terminating.

- No incisal edge - easiest to manage, with minimum change in appearance.

- Cover incisal edge - less stress on internal aspect of veneer if rounded.

- Wrap around incisal edge - used with significant wear \& B-L width is thick. In addition, studies indicate that porcelain is stronger under compression. The wrap-around preparation helps place veneers under compression, hence giving better results. Porcelain under compression is stronger and wrap-around preparation place veneers under compression, thus give a better result (Ref: [12],[14]).

In this case, since there was significant wear observed, wrap around incisal edge was used.

\subsection{Procedure - Impressions}

Retraction cords were placed, and a two-step light body putty impression was recorded for the patient.

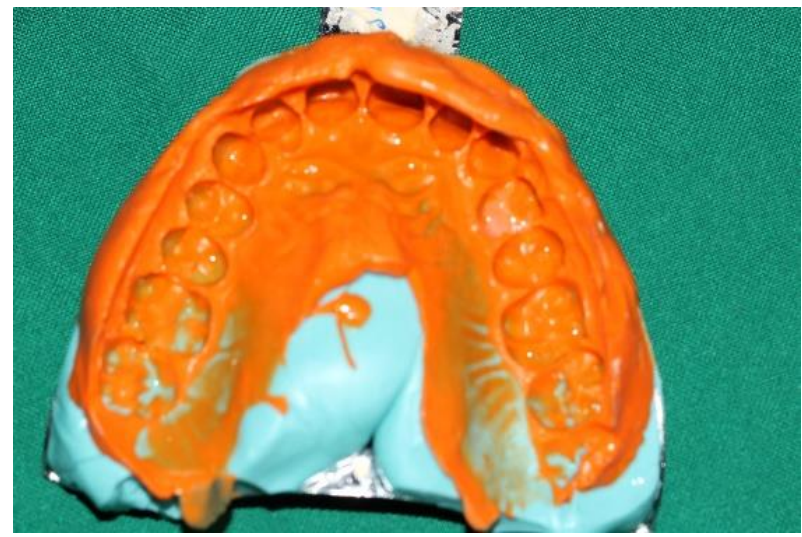

Figure 4: Final Impressions (Case Review)

\subsection{Procedure - Temporization}

Temporization was done as a temporary arrangement while permanent veneers were prepared

- In this case, the shade selected was A1, as the patient insisted on having a whiter shade.

- After the preparations, temporization was done with pro temp (shade : A1). In this case, temporary veneers were given from 13 to 23 by using immediate temporization material (Protemp, 3m espe). 
IJAMSR 3 (4) www.ijamsr.com CrossRef: https://doi.org/10.31426/ijamsr.2020.3.4.3212

\section{International Journal of Advanced Multidisciplinary Scientific Research (IJAMSR) ISSN:2581-4281}

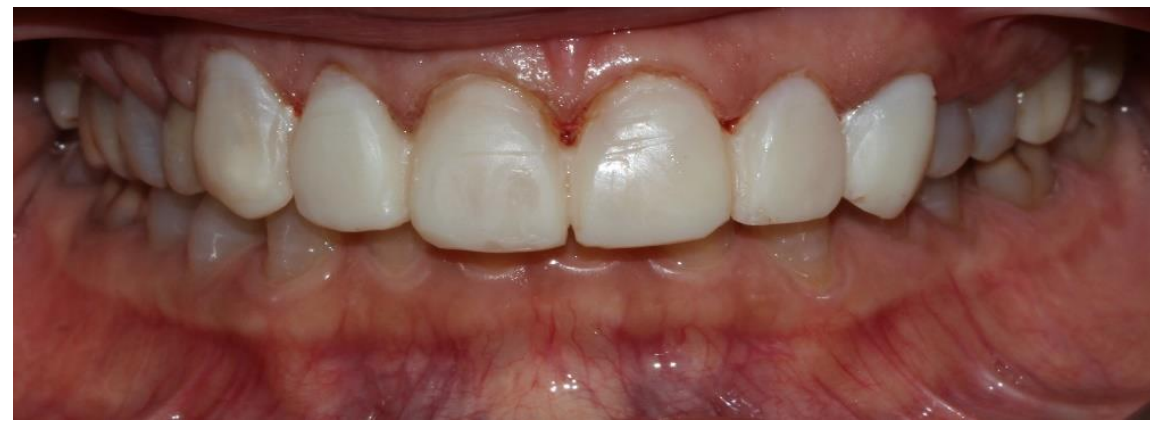

Figure 1: Temporization (Case Review)

\subsection{Procedure - Finalization}

\subsubsection{Finalization: Preparation}

Once the final emax veneers were ready, the patient was called for a try in. After the trial, pictures were taken and once she was satisfied the preparation for final bonding was done.

Preparation of Veneers for Bonding: The steps followed in order to prepare the restoration for final bonding are as below:

- Cleaned the restoration properly with acetone or Cavilax

- Etched the veneers with 10\% Hydro Fluoric Acid \& rinse with water \& airdry.
It is a common clinical practice since it is possible to etch feldspathic porcelain with hydrofluoric acid which gives a great bonding strength to the remaining enamel (Calamia 1982, Nicholls 1988, Stacey 1993, Layton \& Walton 2012) (Ref: [6],[15], [16], [17]).

- Applied a layer of Silane Coupling Agent for 60 seconds and dried with a stream of air (note: do not rinse).

- Left the veneers in lightproof box till ready for bonding.

\section{Preparation of Teeth for Bonding:}

Next, the teeth were prepared for bonding with the veneers. From practice and academic studies, it is important to note that restricting the preparation to enamel is considered to be a critical factor for a favorable bonding strength, thus more durable outcome (Ref: [3], [18]). 
IJAMSR 3 (4) www.ijamsr.com CrossRef: https://doi.org/10.31426/ijamsr.2020.3.4.3212

The steps carried out in preparing the patients' teeth for bonding are listed below:

- Rubber dam isolation

- Cleaning the teeth surfaces with pumice water mixture and rubber cup

- Placing Mylar strip between each tooth

- Etching the teeth with $35 \%$ Phosphoric acid for 15-30 seconds

- Rinsing and air drying; and finally applying the bonding agent.

\subsubsection{Finalization: Bonding}

Once preparations are complete, bonding is done to finalize the treatment.

- The sequence that was followed for bonding was:
a) Both centrals
b) Both Laterals
c) Canines

- Bonding was done by using the $3 \mathrm{M}$ Relyx Resin cement on the veneers and then seating the restorations with firm finger pressure. Excess cement was then removed with a brush.

- After this, each veneer was light cured for 60 seconds from facial to lingual surface.

\subsubsection{Finalization: Finishing \& Polishing}

The procedure is completed with finishing and polishing of the applied veneers

The steps that were followed after bonding the veneers on teeth were:

- Removing excess bonding materials with sharp carver

- Using a floss between each veneer preparation

- Finishing can be initiated with finishing carbides, discs and rubber points.

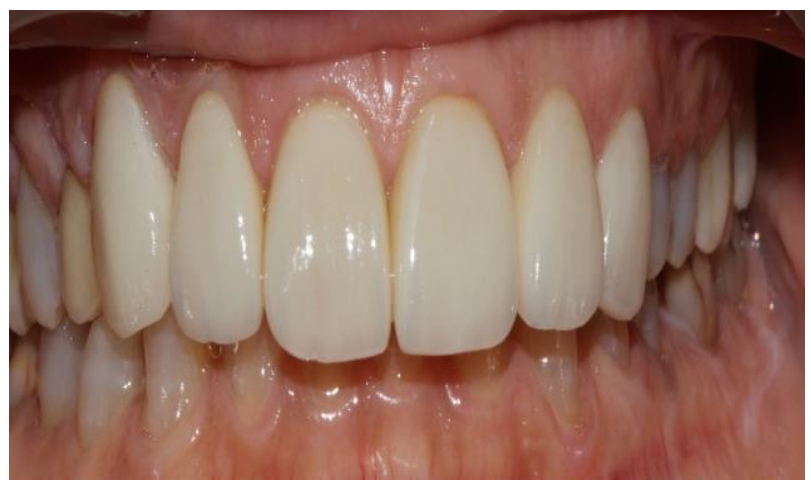

Figure 5: Final Post Operation - Immediate (Case Review) 
IJAMSR 3 (4) www.ijamsr.com CrossRef: https://doi.org/10.31426/ijamsr.2020.3.4.3212

\section{International Journal of \\ Advanced Multidisciplinary Scientific Research (IJAMSR) ISSN:2581-4281}

\section{References}

1) Yousef Alothman, Maryam Saleh Bamasoud The Success of Dental Veneers According To Preparation Design and Material Type. Open Access Maced J Med Sci. 2018 Dec 20; 6(12): 2402-2408. Published online 2018 Dec 14. doi: 10.3889/oamjms.2018.353

2) Brunton, P.A. and Wilson, N.H. Preparations for porcelain laminate veneers in general dental practice. Br Dent J. 1998; 184: 553-556

3) Peumans $M$, et al. Porcelain veneers: a review of the literature. Journal of dentistry. 2000;28(3):163-177. https://doi.org/10.1016/S03005712(99)00066-4

4) Beier, U.S., Kapferer, I., Burtscher, D., and Dumfahrt, H. Clinical performance of porcelain laminate veneers for up to 20 years. Int J Prosthodont. 2012; 25: 79-85

5) Petridis, H.P., Zekeridou, A., Malliari, M., Tortopidis, D., and Koidis, P. Survival of ceramic veneers made of different materials after a minimum follow-up period of five years: a systematic review and meta-analysis. Eur J Esthet Dent. 2012; 7: $138-152$

6) Stacey GD. A shear stress analysis of the bonding of porcelain veneers to enamel. The Journal of prosthetic dentistry. 1993; 70(5):395-402.

https://doi.org/10.1016/0022-

3913(93)90073-W

7) Dr. Ahmed A. Alrashedi. The Veneers Step By Step https://www.slideshare.net/ahmedalrashedi 39/the-veneer-step-by-step

8) Magne P and Belser U. "Bonded porcelain restorations in the anterior dentition: a biomimetic approach". Quintessence publishing company (2002)

9) Alothman Y and Bamasoud MS. "The Success of Dental Veneers According To Preparation Design and Material Type".
Open access Macedonian Journal of Medical Sciences 6.12 (2018): 2402

10) Stappert CF, et al. Longevity and failure load of ceramic veneers with different preparation designs after exposure to masticatory simulation. The Journal of prosthetic dentistry 2005; 94(2):132-139. https://doi.org/10.1016/j.prosdent.2005.05. 023 PMid:16046967

11) Lin $T$, et al. Fracture resistance and marginal discrepancy of porcelain laminate veneers influenced by preparation design and restorative material in vitro. Journal of dentistry. 2012; 40(3):202- 209. https://doi.org/10.1016/j.jdent.2011.12.008 PMid:22198195

12) Hari $M$ and Poovani S. "Porcelain laminate veneers. A review". Journal of Advanced Clinical and Research Insights 4.6 (2017): 187-190

13) Sowmya S., et al. "Esthetics with veneers: A review". International Journal of Dental Health Concerns 1.1 (2015): 1-5

14) Salah A Yousief., et al. "Types of Veneers in Dental World". EC Dental Science 19.2 (2020): 01-06

15) Layton DM, Walton TR. The up to 21-year clinical outcome and survival of feldspathic porcelain veneers: accounting for clustering. The International journal of prosthodontics. 2012; 25(6): 604-612. PMid:23101040

16) Calamia JR. Etched porcelain facial veneers: a new treatment modality based on scientific and clinical evidence. The New York journal of dentistry. 1982; 53(6): 255-259.

17) Nicholls J. Tensile bond of resin cements to porcelain veneers. The Journal of prosthetic dentistry. 1988; 60(4): 443-447. https://doi.org/10.1016/00223913(88)90245-4

18) Rufenacht CR, Berger RP. Fundamentals of esthetics. first ed. Quintessence Chicago; 1990. 